\title{
Structure and functions of lampbrush chromosomes
}

\author{
KatARZYNa ANDRASZEK *, ElżBIETa SMALEC \\ Department of Animal Genetics and Horse Breeding, Siedlce University of Natural Sciences and Humanities, Siedlce, Poland \\ *Corresponding author: andrasz@uph.siedlce.pl
}

\begin{abstract}
Lampbrush Chromosomes (LBCs) are present in the oocytes of birds, lower vertebrata and invertebrates during the prolonged prophase of the first meiotic division. Their name stems from their similarity to bottle brushes. Lampbrush chromosome of the early prophase is a bivalent, made up of two conjugating homologues. The axis of each homologous chromosome is formed by sister chromatids that are differentiated into regions of transcriptionally active and inactive chromatin. Transcription activity of LBCs is observed as a mantle of symmetrically distributed side loops along the chromosome axis. Changes in transcriptional activity are reflected in changes in their morphology. Transcriptional activity of LBCs is directly connected with physiological processes of the body and shows in the morphological structure of the chromosomes. The use of cytogenetic techniques and in situ hybridization have made it possible to identify unique and repeating sequences as well as DNA replication proteins in LBCs. Particularly, interesting prospects are offered by the possibility of using LBCs in studies of transcriptional activity, cytogenetic investigations of karyotype evolution and genome mapping.
\end{abstract}

Key words: meiotic chromosome, lampbrush chromosome, transcription

The progress in biotechnology that was witnessed at the turn of $20^{\text {th }}$ and $21^{\text {st }}$ century was a breakthrough in scientific research - predominantly of experimental nature. The methods and quality of laboratory experiments were improved. A chance also appeared to explain the unverified cytogenetic and molecular genetic issues. The fundamental purpose of genetic research is still to optimally explore the karyotype and genome of animals and plants of various systematic categories and the mechanisms that operate in the cells of those organisms.

Cytogenetic analyses mostly concentrate on somatic cells, particularly blood cells. This is due to the availability of the material which can be sampled in a relatively non-invasive way. The related culture techniques and methods of obtaining preparations of chromosomes caught at the metaphase of the mitotic division have already been well developed. Preparations normally allow the observation of a full set of chromosomes, general analysis of individual karyotypes, and diagnosis of basic aberrations. Cytogenetic research less frequently analyses cells during the meiotic division. Cytogenetic analysis of oocytes is sporadically undertaken, the relevant material being maturing oocytes isolated from the ovaries of animals.
Oocyte development takes place during the meiotic prophase and for the majority of species ends in the metaphase of the first meiotic division. The major part of this development occurs in the diplotene. At that time, the diplotene chromosomes of certain groups of vertebrates, i.a. amphibians and birds, assume the form of lampbrush chromosomes (LBCs) and generate thousands of loops along their axes. The loops are transcriptionally active sites.

\section{History of the term "lampbrush chromosomes"}

Lampbrush chromosomes were discovered in salamander egg cells (Ambystoma mexicanum) by Flemming in 1882. Ten years later, LBCs were identified in shark egg cells and described by Rückert in 1982. It was Rückert who introduced the term "lampbrush chromosome" into biological nomenclature. The chromosomes obtain their name from the $19^{\text {th }}$ century brushes that were used for cleaning street lamps to which Rückert likened them to the same. Modern version of the item is a bottle or test-tube brush (Fig. 1) (Callan, 1986; Macgregor, 1977, 1980, 1987; Macgregor and Varley, 1988). 


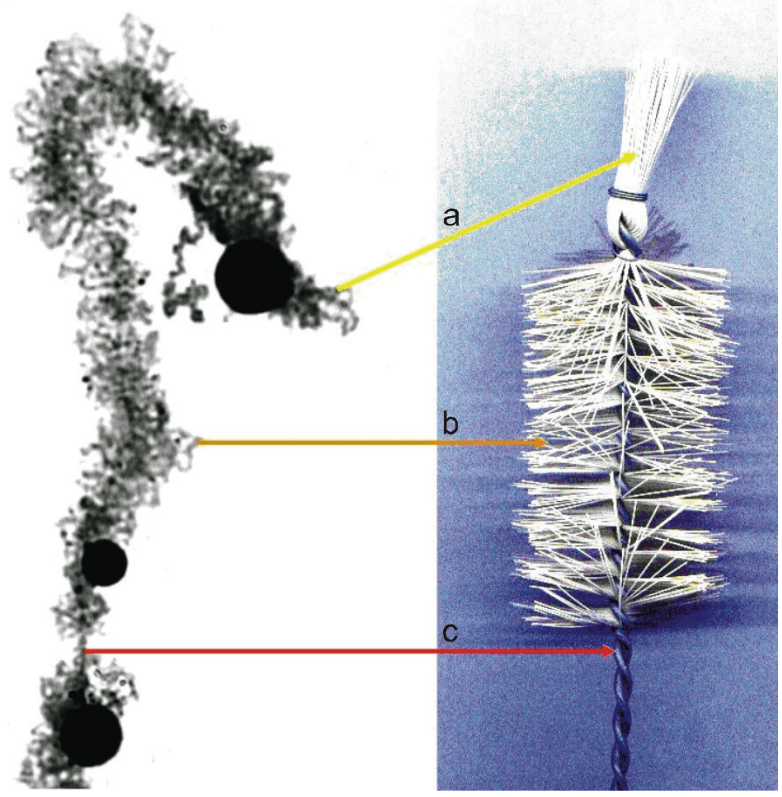

Fig. 1. A lampbrush chromosome and the "original item". The arrows indicate analogous structures; $\mathrm{a}$ - telomeric loop, $\mathrm{b}$ - side loops, c - a chromatid without loops (K. Andraszek, unpublished)

Lampbrush chromosomes are intermediate structures present during the first meiotic division. In a prolonged diplotene stage, they undergo decondensation that results in the production of very large chromosomal structures. LBCs' length ranges (depending on the species) from 400 to $800 \mathrm{~mm}$, which makes them up to 30 times larger than their mitotic counterparts (Callan, 1986; Callan, et al. 1987; Rodionov, 1996).

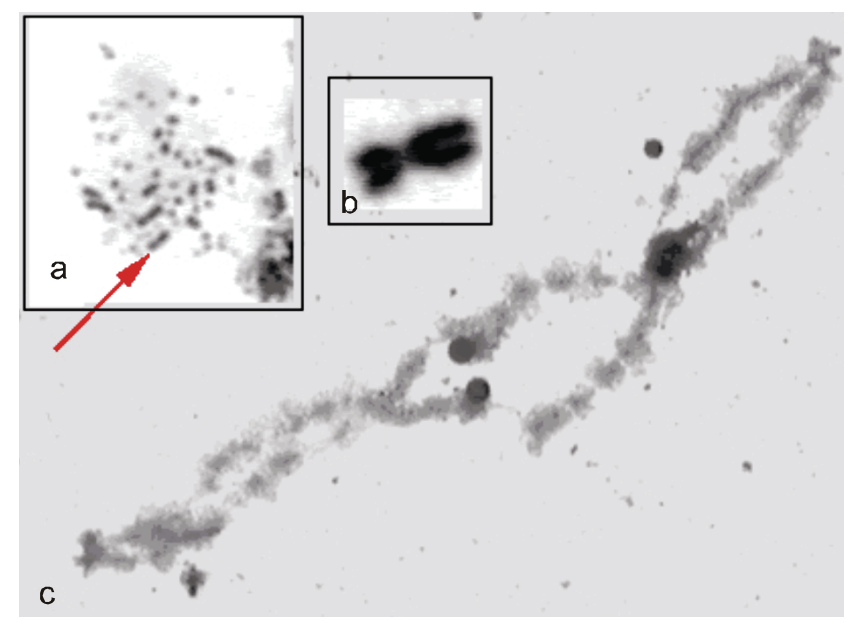

Fig. 2. A comparison of the size of LBCs and mitotic chromosomes. A 20 -fold microscopic magnification of the metaphase plate (a), 100-fold magnification of the second-pair mitotic chromosome (b), a 20-fold magnification of the second lampbrush bivalent (c). The arrow points at the second-pair mitotic chromosome on the metaphase plate (K. Andraszek)
The basic profile of LBCs was performed with a $20 \times$ zoom of the microscope. In the case of avian mitotic chromosomes, a $20 \times$ zoom only makes it possible to identify the metaphase plate, not always enabling the determination of the number of chromosomes. Figure 2 shows a comparison of the proportions of a metaphase plate at a $20 \times$ zoom, the metaphase second-pair chromosome at a $100 \times$ microscopic zoom and the second lampbrush bivalent at a $20 \times$ zoom. The mitotic chromosomes were isolated from peripheral blood of domestic geese. The LBCs, in turn, were sampled from oocytes of domestic geese.

\section{Lampbrush chromosome structure}

In the early prophase, a LBC is a bivalent that consists of two pairs of conjugating homologues, eventually forming a tetrad. Each chromatid is composed of alternately positioned regions of condensed inactive chromatin (chromomeres visible as dark irregular structures and also observed in the interphase nucleus) and side loops of decondensed chromatin. In the homologous sections of the bivalent, chromatin is condensed (spirally twisted) or decondensed in the form of side loops - two per each chromosome and four at the level of the bivalent. The loop constitutes a part of the chromosome axis. It is extensible as well as contractible. The contractibility of the loop results in the contraction and dilation of the chromomere (Angelier, et al. 1984, 1990; Macgregor, 1987; Chelysheva, et al. 1990; Morgan, 2002). A diagram of LBC structure is presented in Figure 3.

Employment of a $100 \times$ zoom to analyse LBC structure has made it possible to observe chromomeres, chiasmata and sister chromatids of each bivalent homologue. An identical zoom used for the analysis of avian mitotic chromosomes enables only the identification of their morphological structure in relation to the first couple of macrochromosome pairs. In the early prophase, the LBC is a bivalent that consists of two conjugating homologues ultimately becoming a tetrad. Figure 4 shows a 20 -fold magnification of the second goose bivalent and its distinctive structures visible with a $100 \times$ zoom. Letters next to the arrows correspond with the respective magnifications in Figure 4. In the case of a structural analysis of male meiotic chromosomes, it is not possible to observe these crucial meiotic cytogenetic features. 

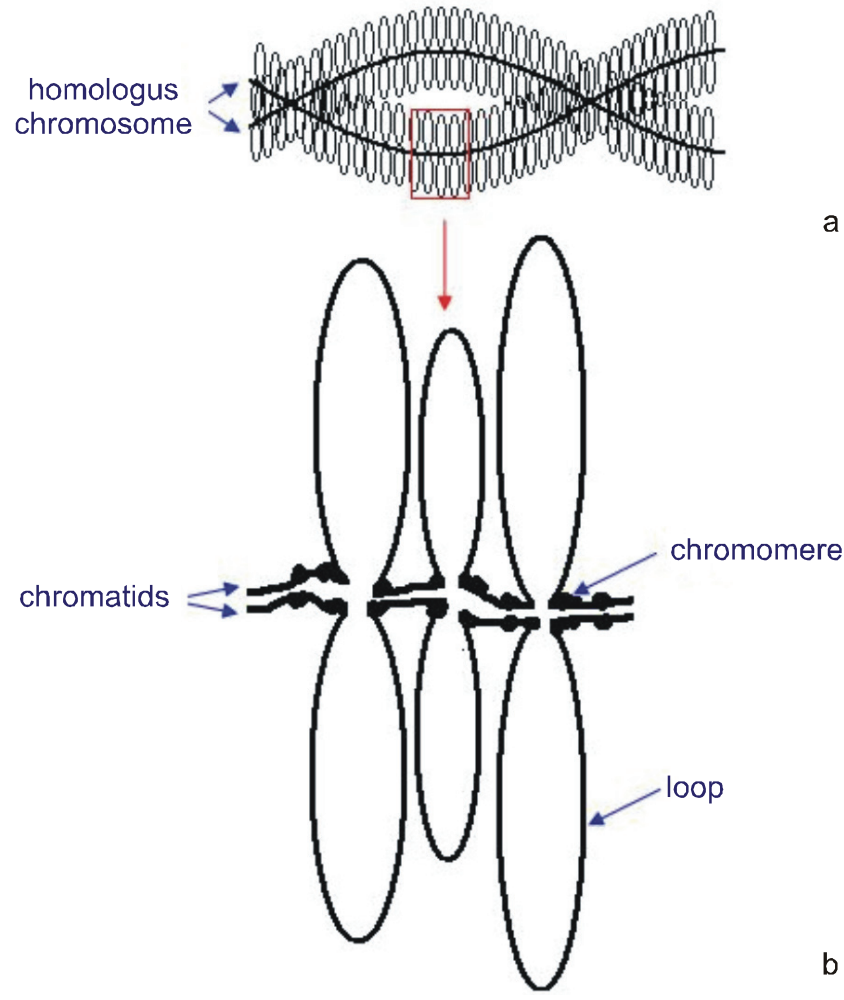

Fig. 3. Schematic (a) and detailed (b) lampbrush chromosome structure (Katarzyna Andraszek)

Numerous morphological types of LBC loops have been identified. Such differentiation is determined by the type and the number of proteins that are directly bound to the emergent transcripts. In terms of transcriptional activity, there are two basic loop types. "Complex" loops have a matrix with a very complicated morphological structure (loop-formed or fibriform). "Complex" loops are classified as marker loops that enable chromosome identification or side loops. Another type of loops are the "plain" loops. They constitute the majority of chromosome loops and have a delicate fibrous matrix, with occasionally well-visible asymmetry. They are always loop-shaped (Angelier et al., 1984; Leòn and Kezer, 1990; Morgan, 2002, 2007).

Lampbrush chromosomes include domains of open chromatin in which the genes can be potentially transcriptive and domains of locked chromatin without expression (Roy et al., 2002; Gaginskaya et al., 2009). Lampbrush chromosome loops are considered an example of open chromatin. Their analogues are thought to be the "puffs" of polytenic chromosomes. They differ between each other. Polytenic chromosomes are made up of parallel chromatids, whereas lampbrush chromosome chromatin constitutes of a single DNA helix. Observation under an electron microscope have demonstrated that the diameter of the loop thread corresponds with the DNA helix diameter, i.e. $1.9 \mathrm{~nm}$ (Olins and Olins, 2003; Gaginskaya et al., 2009).

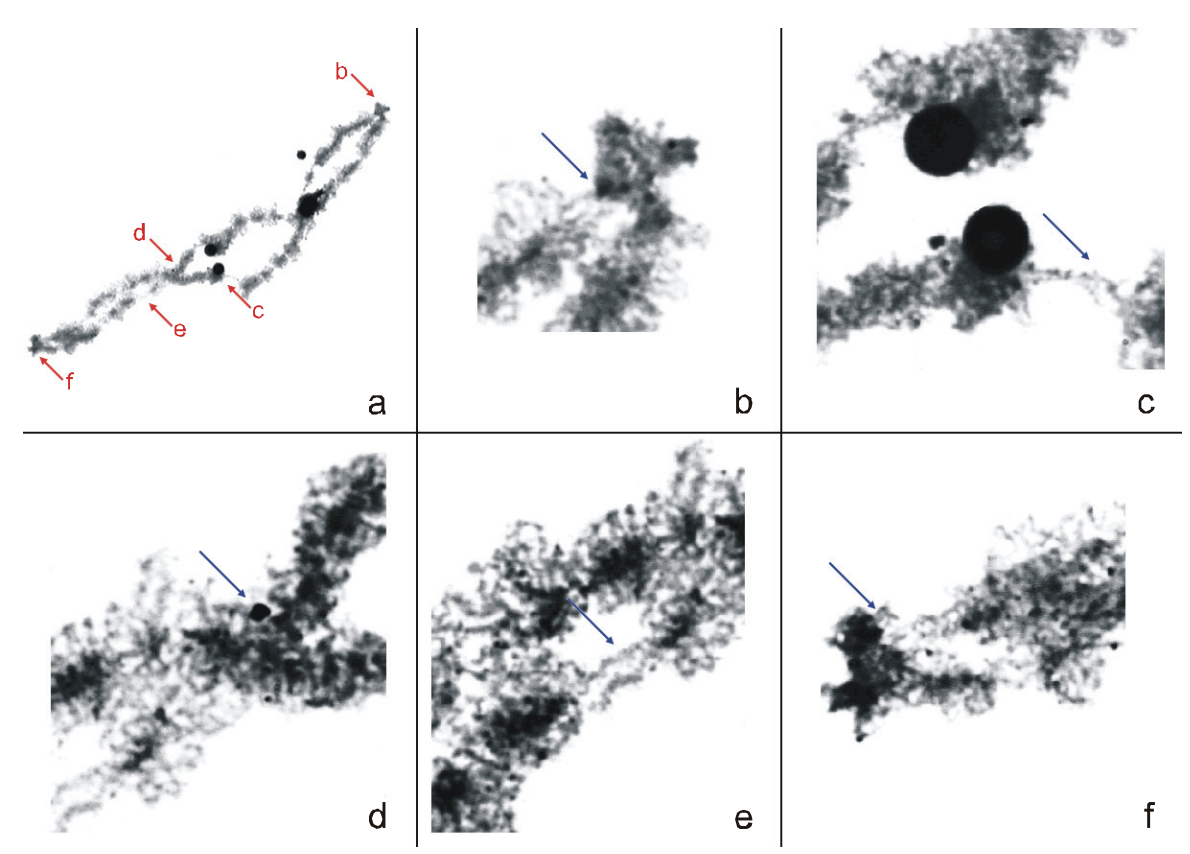

Fig. 4. The second goose lampbrush chromosome with a magnification of its distinctive structures. A 20-fold magnification of the second goose bivalent (a) and its distinctive structures visible with a $100 \times$ zoom (b, f - telomeres, $\mathrm{c}-\mathrm{centromere,} \mathrm{d}-\mathrm{chiasm}$, e - sister chromatids). The particular bivalent structures in the 100-fold blow-ups are marked with blue arrows (K. Andraszek) 
Avian lampbrush chromosomes are associated with protein bodies/structures (PBs). These non-typical structures are present in cells only in association with LBCs. They have a regular connection with the chromosome axis of each LBC in the heterochromatin region (Solovei et al., 1996, Krasikova et al., 2004). In terms of morphology, $\mathrm{PBs}$ resemble Cajal bodies (CBs) present in amphibians in association with LBCs. However, immunocytochemical research has shown that $\mathrm{PBs}$ neither contain $\mathrm{p} 80$ coilin, nor any other $\mathrm{CB}$ matrix indices, such as fibrillarin or splicing- and U7snRNPs-specific trimethyloguanosine epitopes. The distinctive composition of $\mathrm{PBs}$ suggests a completely different function from that of CBs. PBs may be involved in the coordination of spatial layout of chromosomes. The location of PBs is frequently associated with repetitive sequences surrounding the centromere. Exploration of the potential role of centromere-related and centromeric heterochromatin-related proteins in the biogenesis and location of PBs and CBs constitutes a new trend in the research on lampbrush chromosome structure (Gall, 2000; Morgan et al., 2000; Morgan, 2002, 2005; Muphy et al., 2002).

\section{Lampbrush chromosome transcription}

A routine mitotic chromosome analysis can only provide the description of their morphology. Transcriptional activity of genes can only be assessed using molecular methods that consist detecting the amount of the transcription product. Transcriptional activity of LBCs may be observed even under a light microscope and can be determined for morphological changes.

Therefore, LBCs are used as a model in studies of transcriptional regulation. Changes in transcriptional activity result in a different morphological structure of lampbrush chromosome loops (Gall, 1983; Morgan, 2002). Moreover, a higher transcriptional activity of microchromosomes is observed due to a greater density of genes (Rodionov, 1996; Angelier et al., 1984; Morgan, 2002). Transcriptional activity analyses are performed on the basis of assumption that the side loops of LBCs are the transcriptionally active sites. A decrease in transcriptional activity is observed as a shrinking of the side loops (Varley et al., 1980; Gall, 1983; Callan et al., 1987; Gaginskaya and Tsvetkov, 1988; Morgan, 2002, 2007; Galkina et al., 2006; Gaginskaya et al., 2009).

The morphology and transcriptional activity of LBCs vary depending on the reproductive cycle (Andraszek et al., 2009). They can also undergo seasonal changes (Tsvetkov and Parfenov, 1994). This is particularly evident in hibernating amphibians. During the summer, when the animals are the most active, the transcriptional activity of LBCs is the highest, as well. In the autumn, LBCs' activity abates. Nevertheless, this is not associated with morphological changes. At that time of the year, each transcription unit contains approximately $10 \mathrm{RNP}$ (ribonucleoproteinic) filaments, while in the summer, this number is twice as high, the change corresponding with morphological transformation. During the winter, transcription substantially declines. Both in chromosomes and in nucleoli, numerous and very characteristic morphological changes take place (Tsvetkov and Parfenov, 1994). Not more than $70 \%$ of the nuclear DNA is subject to transcription at the time. Although in the case of physical factors, such as radiation or numerous chemical factors, a similar effect on the structure and activity of LBCs in various groups of animals can be expected, seasonal changes predominantly affect polikilotherms (Morgan, 2002, 2007).

The degree of DNA compaction in LBCs is regulated by changes in the distance between nucleosomes, especially the non-adjacent ones. The compaction ratio of DNA (number of DNA $\mu \mathrm{m}$ in a $1 \mu \mathrm{m}$ chromatin fiber) in non-transcribed fibrils is equal to 2.1 , in transcriptional units with moderate and weak activity it is 1.7 , and in transcriptional units with intensive transcription it is close to 1. (Franke and Scheer, 1978; Gaginskaya and Tsvetkov, 1988; Morgan, 2002, 2007). The nucleosomes of transcriptionally inactive chromatin are evenly spaced, the gaps between the nucleosomes corresponding with linker DNA length. In transcription units with insignificant or declining transcription, nucleosomes are identified in the axial part of the chromosome, between sets of polymerase units. The gaps between the polymerase blocks are not even. After polymerase has passed along the DNA matrix and the regulatory proteins have become dissociated, nucleosome reconstruction follows (Spring and Franke, 1981; Gaginskaya and Tsvetkov, 1988; Solovei et al., 1992).

In transcriptionally active regions of chromosomes, histone proteins give way to non-histone proteins, inducing the loss of the nucleosomal structure of the coding chromosome segments that assume the shape of a loop. However, the exposure of nucleosomes, which enables DNA transcription, does not entail histone dissociation 
but leads to a spatial rearrangement of the transcribed regions, allowing access of RNA polymerase to the adjacent promotor sequence. This occurs through the fixation of regulatory proteins at the site of a remote activating sequence. What is particularly the characteristic of lampbrush chromosomes is that non-histone HMG (High Mobility Group) proteins become incorporated in the structure of the chromatin. HMGs are structural proteins of chromatin that reduce chromatin condensation (Di Mario et al., 1989; Korner et al., 2003).

The assumption of appropriate spatial conformation by chromatin enables the commencement of transcription of LBC DNA in the presence of RNA polymerase compounds bound with LBC loops. The transcriptionally active loops represent $5-10 \%$ of DNA. The remainder is inactive chromatin compacted in the chromomeres. The result of transcription is visible under an electron microscope as a ribonucleoproteinic mantle. The mantle tends to be asymmetrical, corresponding with rising electron density from the base towards the middle of the loop (Angelier et al., 1990, 1996).

The average length of a typical lampbrush chromosome loop is $10-15 \mu \mathrm{m}$, though some can be as long as 50 or even $100 \mu \mathrm{m}$. The rate of transcription in lampbrush chromosome loops determined with a radioactive RNA precursor is $5 \mu \mathrm{m}$ per hour. Thus, one loop is transcribed within two to a dozen or so hours. DNA compaction degree in LBCs is not well known. However, it is estimated that $1 \mu \mathrm{m}$ of loop length contains around 3 thousand base pairs. Thus, an average loop contains about 30-40 thousand base pairs, which corresponds with the average length of RNA transcribed in the oocytes. Nevertheless, it is much longer than transcription units of somatic cells. This is the result of skipping transcription termination signals (Kropotova and Gaginskaya, 1984; Hutchison, 1987; Gaginskaya and Tsvetkov, 1988; Morgan, 2002, 2007).

The loops can be classified according to the type of the transcriptional polymerase. The largest loops include those transcribed by polymerase II. The smallest loops are transcribed by polymerase III. They contain 5S RNA coding units (Kay and Gall 1981), tRNA (Müller et al. 1987) or short replication sequences (Kroll et al. 1987). Since 5S RNA sequences are short and divided by noncoding elements, transcription being basically limited to coding sequences, the transcripts of these sequences are also short and, consequently, do not have the dis- tinctive matrix made up of RNP filaments. That is why they are so well visible in the microscopic phase contrast (Murphy et al., 2002). LBCs can be divided into those with one transcriptional unit and those with two or more. Over the length of $1 \mu \mathrm{m}$, one transcriptional unit is transcribed by a densely compacted package of around 13-20 polymerase molecules (Leòn and Kezer, 1990; Macgregor and Varley, 1988; Morgan, 2002).

Regulation of LBC transcription is performed by means of modifications of chromosome structure and the activity of a number of post-transcription factors. The process of transcriptional activity modification consists of a set of interrelated reactions in which numerous interconnected, both structural and enzymatic, factors take part. The first stage is the loosening of chromatin. This is the element that differentiates LBCs from mitotic chromosomes. While somatic cell chromosomes completely lose their structure during transcription, LBCs retain it. The preservation of the structure by LBCs during transcriptional activity is connected with the presence of so-called "constitutive" nucleosomes (Scheer et al., 1984; Scheer, 1987; Gaginskaya and Tsvetkov, 1988; Olins and Olins, 2003). The transcription of the oocytespecific topoisomerase I (topo-I) variant is activated during the formation of LBC structures. This topoisomerase is present in $\mathrm{LBC}$ loops and participates in the spatial conformation of these structures. The inhibition of topo-I activity causes the lampbrush loops to recede and stimulates the condensation of nuclear chromatin (Gebauer et al. 1996). Observations of LBC loops with an electron microscope revealed that the twisting loops contain transcriptive polymerases that are less closely compacted than during active transcription. Additionally, an accompanying condensation of the loops between those polymerases into the form of nucleosomes was observed (Scheer, 1987; Morgan, 2002).

In the condensed segments, the chromomeres build up compact chromatin in which genes or gene-containing transcriptional units are not transcribed. During oogenesis, loops of approximately $50 \mu \mathrm{m}$ in length correspond with active transcriptional units. They constitute $5-10 \%$ of the total length of the chromosome. Chromosome maps of different oocytes at various ages are identical and remain constant for a given oocyte, which suggests a species-specific nature of sequences transcribed during oogenesis. It was possible to identify RNA transcribed in some of the loops and thus initiate the mapping of 
lampbrush chromosomes (Callan, 1986; Chelysheva et al., 1990; Morgan, 2002, 2007; Galkina et al., 2006; Saifitdinova et al., 2003; Gaginskaya et al., 2009).

\section{Lampbrush chromosomes in cytogenetics and genomics}

Lampbrush chromosomes of various species have a very similar structure and perform the same function. Comparative studies of LBCs in various species have shown that the side loops seem to be much longer in species with higher $\mathrm{C}$-values (genome size refers to the haploid set of chromosomes). This regularity explicitly reflects differences in the organisation of genome sequences. One explanation of the effect of genome size on the loop length is based on the existence of substantial differences in the length and distribution of transcribed sequences in relation to chromomere sequences in variously sized genomes (Macgregor, 1980; Gregory, 2002). Another theory suggests that the total increase in the length of loop transcription units results from socalled "over-transcription" of longer intergenic segments present in larger genomes. On the other hand, research by Gall and Murphy (1998) has proved that loop length is species-specific. What is certain, however, is that some organisational characteristics of sequences of large genomes can, to a certain extent, affect the length of transcriptional units of LBCs.

The sequencing of part of the myosin gene of the triton was a proof that much longer introns are present in the triton gene than in the genome of mammals with C-DNA values similar to those of Xenopus (Casimir et al., 1988). However, the data on DNA sequences in large genomes of tailed amphibians are so few that, at present, it is not entirely known how universally any of the sequencing-based explanations can be used to account for the correlation between high C-DNA values and loop length. Uncertain data relating to the loop length point to another important question concerning the structure and function of LBCs - namely: the affinity, organisation and control of transcription loops. The "overtranscription" model initially provided a basis for understanding why in transcriptional units of LBCs there appeared highly repetitive sequences along with pol II complexes containing complexes that initiate transcriptional elongation of the loops. These complexes are assumed to fail to react to termination signals and keep transcribing flanking regions saturated with repetitions (Morgan, 2002, 2007).

Particular interest in recent years has been devoted to possibilities of using LBCs in genome mapping. This strategy can combine chromosome marker mapping and physical gene mapping using the in situ hybridisation technique with genetic maps constructed on the basis of chiasm incidence in the analysed bivalents. Equally important is the possibility of using lampbrush chromosomes in the analyses of the interaction of genes with other cellular structures. Particularly promising seem to be the possibilities of using lampbrush chromosomes in the mapping of avian genomes (Griffin et al., 2088; Penrad-Mobayed et al., 2009; Bi and Bogart, 2010; Daks et al., 2010; Solinhac et al., 2010).

Lampbrush chromosomes were first used as the objects for cytogenetic analyses of poultry by Kropotova and Gaginskaya (1984) and Hutchison (1987). The authors believe that LBCs provide valuable information on the expression of avian genes. They also claim that the chromosomes are indispensable for the cytogenetic study of animals with small genomes, where the large numbers of mitotic chromosomes and their small sizes preclude the analysis of microchromosomes. As in the case of banding patterns of mitotic chromosomes, LBCs have a specific configuration of active and non-active chromomeres observed as a pattern of side loops and loopless areas (Andraszek and Smalec, 2011). In a report on the genome and chromosomes of Gallus domesticus, lampbrush chromosomes were recognised as a new model in avian cytogenetics (Schmid et al., 2005).

Epigenetic mechanisms acting at the level of DNA methylation and histone modification change the structure of LBC chromatin and control the interaction of active and non-active genes. The open conformation of chromatin is transcriptionally active, whereas the "closed" conformation is associated with so-called transcription decline (Grummt and Pikaard, 2003). LBCs are divided into domains containing open chromatin in which the genes can be potentially transcriptive, and domains of locked chromatin without detectable sequence expression. The loops of LBCs are a classic example of an open chromatin. Moreover, oocyte transcription is a complex process in which, apart from LBCs, other nuclear structures are involved as well (Gall, 1983, 2000; Gall et al., 1999; Saifitdinova et al., 2003). 
In conclusion, studies on lampbrush chromosomes have been conducted for over a hundred years. And yet, only a general idea of $\mathrm{LBC}$ structure has been developed thus far. What is not known are the factors that initiate the changes that transform condensed chromosomes into decondensed lampbrush structures. LBCs are considered as model structures in the studies of transcription control. Changes in their transcriptional activity are reflected as modifications of the LBC morphological structure and are associated with the physiological processes of the organism. Moreover, due to their decondensed structure, lampbrush chromosomes are increasingly more often used as objects of cytogenetic analyses, in basic cytogenetic experiments, and as model structures of the epigenetic chromatin control.

\section{References}

Andraszek K., Smalec E. (2011) Comparison of active transcription regions of lampbrush chromosomes with the mitotic chromosome $G$ pattern in the European domestic goose Anser anser. Arch. Tierz. 54: 69-82.

Andraszek K., Smalec E., Tokarska W. (2009) Identification and structure of lampbrush sex bivalents prior to and after the reproduction period of the European domestic goose Anser anser. Folia Biol. (Krakow) 57: 143-148.

Angelier N., Paintraud M., Lavaud A., Lechaire J.P. (1984) Scanning electron microscopy of amphibian lampbrush chromosomes. Chromosoma 89: 243-253.

Angelier N., Bonnanfant-Jais M.L., Herberts C., Lautredou N., Moreau N., N'Da E., Penrad-Mobayed M., RodriguezMartin M.L., Sourrouille P. (1990) Chromosomes of amphibian oocytes as a model for gene expression: significance of lampbrush loops. Int. J. Dev. Biol. 34: 69-80.

Angelier N., Penrad-Mobayed M., Billoud B., BonnanfantJais M.L., Coumailleau P. (1996) What role might lampbrush chromosomes play in maternal gene expression? Int. J. Dev. Biol. 40: 645-652.

Bi K., Bogart J. (2010) Probing the meiotic mechanism of intergenomic exchanges by genomic in situ hybridization on lampbrush chromosomes of unisexual Ambystoma (Amphibia: Caudata). Chromosome Res. 18: 371-382.

Callan H.G. (1986) Lampbrush chromosomes. Springer Verlag, Berlin, Heidelberg, New York, Toronto, 1986.

Callan H.G., Gall J.G., Berg C.A. (1987) The lampbrush chromosomes of Xenopus laevis: preparation, identification, and dis tribution of 5S DNA sequences. Chromosoma 95: 236-250.

Casimir C.M., Gates P.B., Ross-Macdonald P.B., Jackson J.F., Patient R.K., Brockes J.P. (1988) Structure and expression of a new cardio-skeletal myosin gene. Implications for the $C$ value paradox. J. Mol. Biol. 202: 287-296.

Chelysheva L.A., Solovei I.V., Rodionov A.V., Yakovlev A.F., Gaginskaya E.R. (1990) Lampbrush chromosoms of the chicken: the cytological map of the macrobivalents. Cytology 32: 303-316.

Daks A., Derjusheva S., Krasikova A., Zlotina A., Gaginskaya E., Galkina S. (2010) Lampbrush chromosomes of the Japanese quail(Coturnix coturnix japonica): a new version of cytogenetic maps. Rus. J. Genet. 46: 1335-1338.

Di Mario P.J., Bromley S.E., Gall J.G. (1989) DNA-binding proteins on lampbrush chromosome loops. Chromosoma 97: 413-420.

Franke W.W., Scheer U. (1978) Morphology of transcriptional units at different states of activity. Philosoph. Trans. Royal Soc. London 283: 333-342.

Gaginskaya E.R., Tsvetkov A.G. (1988) Electron microscopy research on the chromatin structure of dispersed lampbrush chromosomes in the hen. Tsitologiia 30: 142-150.

Gaginskaya E., Kulikova T., Krasikova A. (2009) Avian lampbrush chromosomes: a powerful tool for exploration of genome expression. Cytogenet. Genome Res. 124: 251-267.

Galkina S., Deryusheva S., Fillon V., Vignal A., Crooijmans R., Groenen M., Rodionov A., Gaginskaya E. (2006) FISH on avian lampbrush chromosomes produces higher resolution gene mapping. Genetica 128: 241-251.

Gall J.M. (1983) Transcription of repetetive sequences on Xenopus lampbrusch chromosomes. Proc. Natl. Acad. Sci. USA 80: 3364-3367.

Gall J.G., Murphy C. (1998) Assembly of lampbrush chromosomes from sperm chromatin. Mol. Biol. 9: 733-747.

Gall J.G., Bellini M., Zheng'an W., Murphy C. (1999) Assembly of the nuclear transcription and processing machinery: Cajal bodies (coiled bodies) and transcriptosomes. Mol. Biol. Cell 10: 4385-4402.

Gall J.G. (2000) Cajal bodies: the first 100 years. Annu. Rev. Cell. Dev. Biol. 6: 273-300.

Gebauer D., Mais C., Zinger K., Hock R., Lieb B., Scheer U. (1996) Localization of a high molecular weight form of DNA topoisomerase I in amphibian oocytes. Int. J. Dev. Biol. 40: 239-244.

Gregory T.R. (2002) A bird's-eye view of the $C$-value enigma: genome size, cell size, and metabolic rate in the class Aves. Int. J. Org. Evolution 56: 121-130.

Griffin D., Robertson L.B., Tempest H.G., Vignal A., Fillon V., Crooijmans R.P.M.A., Groenen M.A.M., Deryusheva S., Gaginskaya E., Carré W., Waddington D., Talbot R., Völker M., Masabanda J.S., Burt D.W. (2008) Whole genome comparative studies between chicken and turkey and their implications for avian genome evolution. BMC Genomics 9: 168 .

Grummt I., Pikaard C.S. (2003) Epigenetic silencing of RNA polymerase I transcription. Nat. Rev. Mol. Cell Biol. 4: 641-649.

Hutchison N. (1987) Lampbrush chromosomes of the chicken Gallus domesticus. J. Cell Sci. 105: 1493-1500.

Kay B.K., Gall J.G. (1981) 5 S ribosomal RNA genes of the new Notophthalmus viridescens. Nucl. Acid Res. 9: 6457-6469.

Korner U., Bustin M., Scheer U., Hock R. (2003) Developmental role of HMGN proteins in Xenopus laevis. Mech. Dev. 120: 1177-1192. 
Krasikova A., Kulikova T., Saifitdinova A., Derjusheva S., Gaginskaya E. (2004) Centromeric protein bodies on avian lampbrush chromosomes contain a protein detectable with an antibody against DNA topoisomerase II. Chromosoma 113: 316-323.

Kroll A., Carbon P., Ebel J.P., Appel B. (1987) Xenopus tropicalis U6 snRNA genes transcribed by Pol III contain the upstream promoter elements used by Pol II dependent $U$-snRNA genes. Nucl. Acids Res. 15: 2463-2478.

Kropotova E.V., Gaginskaya E.R. (1984) Lampbrush chromosomes from the Japanese quail oocytes. Tsitologiia 26: 1008-1015.

Le Moigne A. (1999) Biologia rozwoju. Wydawnictwo Naukowe PWN, Warszawa.

Leòn P., Kezer J. (1990) Loop size in newt lampbrush chromosomes. Chromosoma 99: 83-86.

Macgregor H.C. (1977) Chromatin and chromosome structure. Academic Press.

Macgregor H.C. (1980) Recent developments in the study of lampbrush chromosomes. Heredity 44: 3-35.

Macgregor H.C. (1984) Chromosome structure and function. Van Rostrand i Reinhold Publishing Corp, New York.

Macgregor H.C. (1987) Lampbrush chromosomes. J. Cell Sci. 88: 7-9.

Macgregor H.C., Varley J. (1988) Working with animal chromosomes. John Wiley \& Sons. London, New York, Brisbane, Toronto, Singapore.

Morgan G.T. (2002) Lampbrush chromosomes and associated bodies: new insights into principles of nuclear structure and function. Chromosome Res. 10: 177-200.

Morgan G.T. (2007) Localized co-transcriptional recruitment of the multifunctional RNA-binding protein CELF1 by lampbrush chromosome transcription units. Chromosome Res. 15: 985-1000.

Morgan G.T., Doyle O., Murphy C., Gall J.G. (2000) RNA polymerase II in Cajal bodies of amphibian oocytes. J. Struct. Biol. 129: 258-268.

Murphy C., Wang Z., Roeder R.G., Gall J.G. (2002) RNA polymerase III in Cajal bodies and lampbrush chromosomes of the Xenopus oocyte nucleus. Mol. Biol. Cell 13: 3466-3476.

Müller F., Clarkson S.G., Galas D.J. (1987) Sequence of a $3.18 \mathrm{~kb}$ tandem repeat of Xenopus laevis DNA containing 8 tRNA genes. Nucl. Acid Res. 15: 7191.

Olins D.E., Olins A.L. (2003) Chromatin history: our view from the bridge. Nature Rev. Mol. Cell Biol. 4: 809-814.

Penrad-Mobayed M., El Jamil A., Kanhoush R., Perrin C. (2009) Working map of the lampbrush chromosomes or Xenopus tropicalis: a new tool for cytogenetic analysis. Dev. Dyn. 238: 1492-1501.
Rodionov A.V. (1996) Micro versus macro: a review of structure and functions of avian micro- and macrochromosomes. Genetika 32: 597-608.

Roy J.P., Stuart J.M., Lund J., Kim S.K. (2002) Chromosomal clustering of muscle-expressed genes in Caenorhabditis elegans. Nature 418: 975-979.

Saifitdinova A., Derjusheva S., Krasikova A., Gaginskaya E. (2003) Lampbrush chromosomes of the chaffinch (Fringilla coelebs L.). Chromosome Res. 11: 99-113.

Scheer U. (1987) Structure of lampbrush chromosome loops during different states of transcriptional activity as visualized in the presence of physiological salt concentrations. Biol. Cell 59: 33-42.

Scheer U., Hinssen H., Franke W.W., Jockusch B.M. (1984) Microinjection of actinbinding proteins and actin antibodies demonstrates involvement of nuclear actin in transcription of lampbrush chromosomes. Cell 39: 111-122.

Schmid M., Nanda I., Hoehn H., Schartl M., Haaf T., Buerstedde J.M., Arakawa H., Caldwell R.B., Weigend S., Burt D.W. et al. (2005) Second report on chicken genes and chromosomes 2005. Cytogenet. Genome Res. 109: 415-479.

Solinhac R., Leroux S., Galkina S., Chazara O., Feve K., Vignoles F., Morisson M., Derjusheva S., Bed'hom B., Vignal A., Fillon V., Pitel F. (2010) Integrative mapping analysis of chicken microchromosome 16 organization. BMC Genomics 11: 616.

Solovei I.V., Gaginskaya E., Allen T., Macgregor H.C. (1992) A novel structure associated with lampbrush chromosomes in the chicken Gallus domesticus. J. Cell Sci. 101: 759-772.

Solovei I.V., Joffe B.I., Gaginskaya E.R., Macgregor H.C. (1996) Transcription of lampbrush chromosomes of a centromerically localized highly repeated DNA in pigeon (Columba) relates to sequence arrangement. Chromosome Res. 4: 588-603.

Spring H., Franke W.W. (1981) Transcriptionally active chromatin in loops of lampbrush chromosomes at physiological salt concentrations as revealed by electron microscopy of sections. Eur. J. Cell Biol. 24: 298-308.

Tsvetkov A.G., Parfenov V.N. (1994) Seasonal transformations in the lampbrush chromosomes and the morphogenesis of the karyosphere capsule in Rana temporaria oocytes detectable by an analysis of the isolated nuclear structures. Tsitologiia 36: 64-70.

Varley J.M., Macgregor H.C., Erba H.P. (1980) Satelite DNA is transcribed on lampbrush chromosomes. Nature 283: 686-688. 